\title{
SOME ASPECTS OF COVERING THEORY
}

WOODROW W. BLEDSOE AND A. P. MORSE

1. Introduction. We shall understand $M$ is A. P. Morse's paper $A$ theory of covering and differentiation which appeared in Trans. Amer. Math. Soc. vol. 55 (1944) pp. 205-235. We shall understand $\mathrm{M}^{\prime}$ is A. P. Morse's paper Perfect blankets which appeared in Trans. Amer. Math. Soc. vol. 61 (1947) pp. 418-442.

1.0 Definitions.

$.1 \sim B=$ the complement of $B$.

Thus

$$
\sim B=\mathrm{E} x(x \in B)
$$

$=$ the set of points $x$ such that $x$ does not belong to $B$.

.2 sng $y=\operatorname{Ex}(x=y)$.

Thus sng $y$ is the set whose sole member is $y$.

1.1 Definitions.

$.1 \sigma \mathfrak{F}=\mathrm{U}_{\beta \in \mathfrak{F}} \beta$.

$.2 \pi \mathfrak{F}=\bigcap_{\beta \in \mathfrak{F}} \beta$.

Thus $x \in \sigma \mathfrak{F}$ if and only if $x$ is a member of some member of $\mathfrak{F}$, and $x \in \pi \mathfrak{F}$ if and only if $x$ is a member of every member of $\mathfrak{F}$.

1.2 Definition. $\phi$ measures $S$ if and only if $\phi$ is such a function on the subsets of $S$ that: $0 \leqq \phi(A)$ whenever $A \subset S$; and

$$
\phi(A) \leqq \sum_{\beta \in \Im} \phi(\beta)
$$

whenever $\mathfrak{F}$ is a countable family for which $A \subset \sigma \mathfrak{F} \subset S$.

Clearly if $\phi$ measures $S$ and $\phi$ measures $T$, then $S=T$.

1.3 Definition. $\phi$ is a measure if and only if $\phi$ measures some set.

1.4 Definition. A set $A$ is $\phi$ measurable if and only if $\phi$ measures some superset $S$ of $A$ in such a way that

$$
\phi(T)=\phi(T A)+\phi(T \sim A)
$$

whenever $T \subset S$.

1.5 Definition. $\mathfrak{F}$ has the $\phi$ Vitali property with respect to $A$ if and only if $\phi$ is a measure and $\mathfrak{F}$ is such a family of $\phi$ measurable sets that corresponding to each finite disjointed subfamily $\mathfrak{S}$ of $\mathfrak{F}$ there is a countable disjointed family (S) for which

$$
\mathfrak{S} \subset \mathcal{S} \subset \mathfrak{F} \text { and } \phi(A \sim \sigma(\mathfrak{S})=0 \text {. }
$$

1.6 Definition. $\mathfrak{F}$ is Vitalian with respect to $A$ if and only if corresponding to each finite disjointed subfamily $\mathfrak{S}$ of $\mathfrak{F}$ there is a

Received by the editors November 19, 1951. 
countable disjointed family of $\&$ for which

$$
\mathfrak{S} \subset \mathbb{S} \subset \mathfrak{F} \text { and } A \subset \sigma(s)
$$

Almost obvious is the following theorem.

1.7 THEOREM. If $A \subset \sigma \mathfrak{F}$ and $\phi$ measures $\sigma \mathfrak{F}$ in such a way that $\phi(\beta)=$ the number of points in $\beta$ whenever $\beta \subset \sigma \mathfrak{F}$, then $\mathfrak{F}$ is Vitalian with respect to $A$ if and only if $\mathfrak{F}$ has the $\phi$ Vitali property with respect to A.

A consequence of Theorem 5.2 and $\$ 2$ of $\mathrm{M}$ is the following theorem.

1.8 THEOREM. If $\Omega$ is such a nonvacuous countable family of sets of finite $\phi$ measure that $\mathfrak{F}$ has the $\phi$ Vitali property with respect to each member of $\Omega$, then $\mathfrak{F}$ has the $\phi$ Vitali property with respect to $\sigma \Omega$.

From this we see at once the following theorem.

1.9 THEOREM. If

$$
A=\bigcup_{n=1}^{\infty} S(n)
$$

and if, for each positive integer $n, S(n)$ has finite $\phi$ measure and the family $\mathfrak{F}$ has the $\phi$ Vitali property with respect to $S(n)$, then $\mathfrak{F}$ has the $\phi$ Vitali property with respect to $A$.

A. P. Morse negligently recast Theorem 5.2 of $\mathrm{M}$ as Theorem 3.2 of $\mathrm{M}^{\prime}$. As stated both 3.2 and 3.3 of $\mathrm{M}^{\prime}$ are false since also false is the following:

1.10. Statement. If $\mathfrak{F}$ is Vitalian with respect to $A^{\prime}$ and $\mathfrak{F}$ is Vitalian with respect to $A^{\prime \prime}$, then $\mathfrak{F}$ is Vitalian with respect to $A^{\prime} \cup A^{\prime \prime}$.

We shall construct a counterexample which makes it easy to settle a related problem in blanket theory. Our counterexample is based on a simple preliminary theory which, we feel, is of some interest in itself.

In order to correct the situation arising from the erroneous formulation of Theorems 3.2 and 3.3 of $\mathrm{M}^{\prime}$ simply replace them respectively by Theorems 1.8 and 1.9 of the present paper, and then replace formula (1) in the proof of Theorem 5.11 of $\mathrm{M}^{\prime}$ by the following:

1.11 FORMULA

$$
S_{\lambda}=S(\theta, \lambda) \underset{x}{\mathrm{E}}\left[\operatorname{limalsup}_{F(x) \ni \beta \rightarrow x} \frac{\operatorname{diam} \beta}{\operatorname{hbrd}(x, \beta)}<\lambda\right] .
$$

We should also like to suggest that Theorem 2.19 of $\mathrm{M}^{\prime}$ be replaced by: 
1.12 Theorem. If $F$ is a perfect blanket with domain $\mathcal{S}, f \in \mathcal{U}$, $\phi \in \mathscr{W}$

$$
B=\underset{x}{\mathrm{E}}(\boldsymbol{D}(f, f+\phi, F, x)=1)
$$

then a necessary and sufficient condition that $f$ be $\phi$ conservative is that $f(B)=0$.

As stated Theorem 2.19 of $\mathrm{M}^{\prime}$ is valid if and only if $a / 0=\infty$ whenever $a>0$.

Furthermore, definition $12.1 \mathrm{M}$ of comparative regularity is unsound. One simple way of correcting it is to add to $11.1 \mathrm{M}$ the condition that diam $\alpha \leqq \lambda$ diam $\beta$.

We include 2.1-2.12 below for convenience and completeness. The concepts involved have already been examined by others in somewhat different formulations. See, for example, G. Kurepa, Ensembles ordonnées et ramifiés, Publications Mathématiques de l'Université de Belgrade, vol. 4, 1935, especially pp. 67-74.

2. Nets.

2.1 Definition. $\mathfrak{F}$ is a net if and only if $\sigma(S \in B)$ whenever $\mathbb{B}$ is such a nonvacuous subfamily of $\mathfrak{F}$ that $\pi(\xi \neq 0$.

2.2 Definition. $\mathfrak{F}$ is a $n e t^{\prime}$ if and only if $\mathfrak{F}$ is a net and every disjointed subfamily of $\mathfrak{F}$ is countable.

2.3 Definition. $\mathfrak{F} \subset \subset \mathcal{G}$ if and only if every member of $\mathfrak{F}$ is a subset of some member of $\$$.

Thus $\mathfrak{F} \subset \subset \mathcal{H}$ if and only if $\mathfrak{F}$ is a refinement of $\mathfrak{B}$.

2.4 Definition. $\omega=$ the set of all non-negative integers.

2.5 Theorem. If $A$ is such a sequence of disjointed families that $A(n+1) \subset \subset A(n)$ whenever $n \in \omega$, then

$$
\bigcup_{n \in \omega} A(n)
$$

is a net.

2.6 Theorem. If $\mathfrak{F}$ is a net, then $\beta \subset \beta^{\prime}$ or $\beta^{\prime} \subset \beta$ whenever $\beta$ and $\beta^{\prime}$ are such members of $\mathfrak{F}$ that $\beta \beta^{\prime} \neq 0$.

Proof. Let $\left(H=E \alpha\left(\alpha=\beta\right.\right.$ or $\left.\alpha=\beta^{\prime}\right)$. Since $\pi(H)=\beta \beta^{\prime} \neq 0$, we infer from 2.1 that $\beta \cup \beta^{\prime}=\sigma \leftrightarrow \in \mathcal{B S}$. Thus: $\beta \cup \beta^{\prime}=\beta$ or $\beta \cup \beta^{\prime}=\beta^{\prime} ; \beta \subset \beta$ or $\beta \subset \beta^{\prime}$.

2.7 Theorem. Any subfamily of a net is a net.

2.8 Theorem. Any subfamily of a net' is a net'. 
2.9 ThEOREMS.

.1 If $\mathfrak{F}^{\prime} \subset \mathfrak{F}$, then $\mathfrak{F}^{\prime} \subset \subset \mathfrak{F}$.

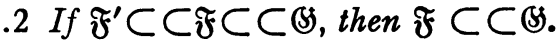

.3 If $\mathfrak{F}^{\prime} \subset \mathfrak{F} \subset \subset \mathrm{S}$, then $\mathfrak{F}^{\prime}=\subset \mathrm{S}$.

.4 If $\mathfrak{F}^{\prime} \subset \subset \mathfrak{F}$, then $\sigma \mathfrak{F}^{\prime} \subset \sigma \mathfrak{F}$.

2.10 Definition. top $\mathfrak{F}=\mathfrak{F E} \beta$ (for each $\alpha, \beta \subset \alpha \in \mathfrak{F}$ implies $\beta=\alpha$ ).

2.11 TheOREM. If $\mathfrak{F}$ is a net, $0 \neq \beta \in \mathfrak{F}$ and $\$=\mathrm{E} \alpha(\beta \subset \alpha \in \mathfrak{F})$, then

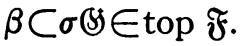

Proof. Since $0 \neq \beta=\pi(S)$, it follows from 2.1 that $\sigma(\mathfrak{S} \in \mathbb{S} \subset \mathfrak{F}$; furthermore, if $\sigma(\mathfrak{S} \subset \alpha \in \mathfrak{F}$, then $\beta \subset \sigma(B) \subset \alpha, \beta \subset \alpha \in \mathfrak{F}, \alpha \in \mathbb{S}, \alpha \subset \sigma \mathfrak{S}, \alpha=\sigma \mathbb{S}$.

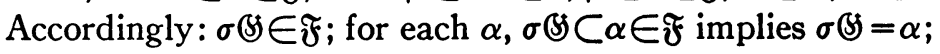

$$
\sigma(s) \in \operatorname{top} \mathfrak{F}
$$

Moreover $\beta \subset \beta \in \mathfrak{F}, \beta \in \$$,

$$
\beta \subset \sigma \text { (s). }
$$

The proof is complete.

2.12 Theorem. If $\mathfrak{F}$ is a net, then:

.1 top $\mathfrak{F}$ is disjointed;

$.2 \mathfrak{F} \subset \subset$ top $\mathfrak{F}$;

$.3 \sigma$ top $\mathfrak{F}=\sigma \mathfrak{F}$.

We divide the proof into 6 parts, the first of which is an immediate consequence of 2.10 .

PART I. If $\alpha \in$ top $F, \beta \in F$, and $\alpha \subset \beta$, then $\alpha=\beta$.

PART II. top $\mathfrak{F}$ is disjointed.

Proof. Suppose $\alpha \in$ top $\mathfrak{F}$ and $\beta \in$ top $\mathfrak{F}$. From 2.6 and the fact that top $\mathfrak{F} \subset \mathfrak{F}$ it follows that

$$
\alpha \beta=0 \text { or } \alpha \subset \beta \text { or } \beta \subset \alpha \text {. }
$$

Thus because of part I

$$
\alpha \beta=0 \text { or } \alpha=\beta \text {. }
$$

PART III. If $\mathfrak{F} \neq 0$, then top $\mathfrak{F} \neq 0$.

PRoOF. If $\mathfrak{F}=$ sng 0 , then top $\mathfrak{F}=$ sng $0=\mathfrak{F} \neq 0$. If $\mathfrak{F} \neq$ sng 0 , then so choose $\beta$ that $0 \neq \beta \in \mathfrak{F}$, let $\mathbb{H}=\mathrm{E} \alpha(\beta \subset \alpha \in \mathfrak{F})$, and use 2.11 to infer that $\sigma(B)$ top $\mathfrak{F} \neq 0$.

PART IV. If $\beta \in F$, then there is a set $\gamma$ for which $\beta \subset \gamma \in$ top $\mathfrak{F}$.

Proof. If $\beta \neq 0$, then let $\mathbb{S}=\mathrm{E} \alpha(\beta \subset \alpha \in \mathfrak{F})$, take $\gamma=\sigma(B)$, and apply 2.11 . 
If $\beta=0$, then in accordance with part III choose $\gamma$ so that $\beta=0$ $\subset \gamma \in$ top $\mathfrak{F}$.

PART V. $\mathfrak{F} \subset \subset$ top $\mathfrak{F}$.

Proof. Apply part IV.

PART VI. $\sigma$ top $\mathfrak{F}=\sigma \mathfrak{F}$.

Proof. Since top $\mathfrak{F} \subset \mathfrak{F}$, it is clear that $\sigma$ top $\mathfrak{F} \subset \sigma \mathfrak{F}$. Furthermore, it follows from part $\mathrm{V}$ and 2.9.4 that $\sigma \mathfrak{F} \subset \sigma$ top $\mathfrak{F}$. Thus $\sigma$ top $\mathfrak{F}=\sigma \mathfrak{F}$.

2.13 THEOREM. If $\mathfrak{F}$ is a net', then:

.1 top $\mathfrak{F}$ is disjointed and countable;

$.2 \mathfrak{F} \subset \subset$ top $\mathfrak{F}$

$.3 \sigma$ top $\mathfrak{F}=\sigma \mathfrak{F}$.

Proof. Use 2.2 and 2.12 .

2.14 Definition. $\mathfrak{F}^{\prime}$ enmeshes $A$ with respect to $\mathfrak{F}$ if and only if $\mathfrak{F}^{\prime}$ is such a net' that for each $\alpha \in \mathfrak{F}$ and each $x \in A \sim \alpha$ there corresponds a member $\beta$ of $\mathfrak{F}^{\prime}$ for which $x \in \beta$ and $\alpha \beta=0$.

2.15 Theorem. If $\mathfrak{F}^{\prime}$ enmeshes $A$ with respect to $\mathbb{B}$ and $\mathfrak{F} \subset(B)$, then $\mathfrak{F}^{\prime}$ enmeshes $A$ with respect to $\mathfrak{F}$.

It is well known that $\pi \Omega \in \Omega$ whenever $\Omega$ is such a finite nonvacuous family of sets that $\alpha \subset \beta$ or $\beta \subset \alpha$ whenever $\alpha \in \Omega$ and $\beta \in \Omega$.

A consequence is the following:

2.16 LemMa. If $\mathfrak{F}^{\prime}$ is a net and $\Re$ is such a finite subfamily of $\mathfrak{F}^{\prime}$ that $\pi \Omega \neq 0$, then $\pi \Re \in \Omega$.

2.17 Lemma. If $\mathfrak{F}^{\prime}$ enmeshes $A$ with respect to $\mathfrak{F}, \mathfrak{S}$ is a finite subfamily of $\mathfrak{F}$, and $x \in A \sim \sigma \mathfrak{S}$, then there is such a member $\beta$ of $\mathfrak{F}^{\prime}$ that $x \in \beta$ and $\beta \sigma \mathfrak{S}=0$.

Proof. Apply 2.14 and 2.16.

2.18 THEOREM. If $\mathfrak{F}^{\prime} \subset \mathfrak{F}$ and $\mathfrak{F}^{\prime}$ enmeshes $A$ with respect to $\mathfrak{F}$, then F is Vitalian with respect to $A$.

Proof. Let $\mathfrak{S}$ be a finite disjointed subfamily of $\mathfrak{F}$. Helped by 2.17 we choose such a function $\beta$ that

$$
x \in \beta(x) \in \mathfrak{F}^{\prime} \text { and } \beta(x) \cap \sigma \mathfrak{S}=0
$$

whenever $x \in A \sim \sigma \mathfrak{S}$.

Let $\mathfrak{F}^{\prime \prime}$ be the family of sets of the form $\beta(x)$ where $x \in A \sim \sigma \mathfrak{G}$ and note that

$$
A \sim \sigma \mathfrak{S} \subset \sigma \mathfrak{F}^{\prime \prime} \subset \sim \sigma \mathfrak{S} .
$$


After checking that $\mathfrak{F}^{\prime \prime} \subset \mathfrak{F}^{\prime} \subset \mathfrak{F}$, infer from 2.8 that $\mathfrak{F}^{\prime \prime}$ is a net' and let .2

$$
\mathbb{B}=\mathfrak{S} \cup \text { top } \mathfrak{F}^{\prime \prime} \text {. }
$$

From .1, 2.13.3, and .2 it follows that

$$
\begin{aligned}
A & \subset \sigma \mathfrak{S} \cup A \sim \sigma \mathfrak{S} \subset \sigma \mathfrak{S} \cup \sigma \mathfrak{F}^{\prime \prime} \\
& =\sigma \mathfrak{S} \cup \sigma \text { top } \mathfrak{F}^{\prime \prime}=\sigma\left(\mathfrak{S} \cup \text { top } \mathfrak{F}^{\prime \prime}\right) \\
& =\sigma \mathfrak{S} .
\end{aligned}
$$

Thus $A \subset \sigma B$. That $\mathfrak{S} \subset \mathcal{B}$ is a consequence of .2 . That $\mathbb{S}$ is a countable disjointed subfamily of $\mathfrak{F}$ is a consequence of 2.13.1 and .1. Appeal to 1.6 completes the proof.

\section{Families of intervals.}

\subsection{Definitions}

$.1 \omega^{\prime}=$ the set of all integers;

$.2 R=$ the set of all real finite numbers;

$.3 \Re=$ the set of all closed intervals in $R$ with finite positive diameters;

$.4 \mathfrak{E}=$ the set of all closed subsets of $R$.

\subsection{Definitions.}

.1 component $A=\mathrm{E} C\left(C \subset A, C\right.$ is connected, and $C^{\prime} \subset C$ whenever $C^{\prime}$ is such a connected subset of $A$ that $\left.C C^{\prime} \neq 0\right)$;

.2 komponent $A=\mathrm{E} C$ (for some $C^{\prime} \in$ component $A, C=$ closure $\left.C^{\prime}\right)$.

3.3 Theorem. If $\beta$ is a connected subset of $R$, diameter $\beta>0$, and (B) is a countable disjointed subfamily of $\mathbb{E}$, then either $\beta \sim \sigma(B)$ has the power of the continuum or $\beta$ is contained in some member of $\mathbb{B}$.

3.4 TheOREM. If $\mathfrak{F}=\mathfrak{F}^{\prime} \cup \mathfrak{F}^{\prime \prime} \subset \mathfrak{N}, \mathfrak{F}^{\prime}$ enmeshes $A^{\prime}$ with respect to $\mathfrak{F}, \mathfrak{F}^{\prime \prime}$ enmeshes $A^{\prime \prime}$ with respect to $\mathfrak{F}$, and $A^{\prime} \cup A^{\prime \prime}=R$, then $\mathfrak{F}$ is Vitalian with respect to $A^{\prime}$ and $\mathfrak{F}$ is Vitalian with respect to $A^{\prime \prime}$ but $\mathfrak{F}$ is not Vitalian with respect to $A^{\prime} \cup A^{\prime \prime}$.

Proof. Apply 2.18, 3.3, and 1.6.

\subsection{Theorem. If $A \subset B \subset R$, then:}

.1 component $A \subset \subset$ component $B$;

.2 komponent $A \subset \subset$ komponent $B$;

.3 component $A$ is disjointed;

$.4 A=\sigma$ component $A \subset \sigma$ komponent $A$.

3.6 LemмA. If $S$ and $T$ are descending sequences of sets, then

$$
\bigcap_{n \in \omega}(S(n) \cup T(n))=\bigcap_{n \in \omega} S(n) \cup \bigcap_{n \in \omega} T(n) .
$$


3.7 Lemma. If $P^{\prime}$ is a perfect subset of $R$, then komponent $\left(R \sim P^{\prime}\right)$ is a countable disjointed subset of $\mathfrak{R}$.

\subsection{THEOREM. If}

$.1 P$ is an ascending sequence of perfect sets;

$.2 Q$ is such $a$ sequence that, for $n \in \omega, Q(n)=$ komponent $(R \sim P(n))$;

$.3 \mathfrak{E}=U_{n \in \omega} Q(n)$;

$.4 A=\bigcap_{n \in \omega} \sigma Q(n)$;

then

$.5 \mathfrak{S}$ is a countable net;

.6 if inf $P(0)=-\infty$ and sup $P(0)=\infty$, then $\mathfrak{S} \subset \mathfrak{N}$;

.7 if $U_{n \in \omega} P(n)$ is dense in $R$, then $\mathfrak{Q}$ enmeshes $A$ with respect to $\mathfrak{G}$

.8 if $n \in \omega$, then $\sigma Q(n+1) \subset \sigma Q(n)$.

3.9 Remarks. Suppose $P^{\prime}$ is such a sequence that, for $n \in \omega, P^{\prime}(n)$ is the set of numbers of the form

$$
\frac{1}{5^{n}}\left\{N+\sum_{j} \in_{\omega} \frac{\alpha(j)}{5^{j+1}}\right\}
$$

where $N \in \omega^{\prime}$ and $\alpha$ is a function on $\omega$ to sng $0 \cup$ sng 1 . Similarly suppose $P^{\prime \prime}$ is such a sequence that, for $n \in \omega, P^{\prime \prime}(n)$ is the set of numbers of the form

$$
\frac{1}{5^{n}}\left\{N+\sum_{j} \in_{\omega} \frac{\alpha(j)}{5^{j+1}}\right\}
$$

where $N \in \omega^{\prime}$ and $\alpha$ is a function on $\omega$ to sng $2 \cup$ sng 3 . Check that $P^{\prime}$ and $P^{\prime \prime}$ are such ascending sequences of perfect sets that:

.1

$$
\begin{aligned}
& \inf P^{\prime}(0)=\inf P^{\prime \prime}(0)^{n}=-\infty ; \\
& \text { sup } P^{\prime}(0)=\sup P^{\prime \prime}(0)=\infty ; \\
& \text { if } n \in \omega \text { then } P^{\prime}(n) \cap P^{\prime \prime}(n)=0 ; \\
& \bigcup_{n \in \omega} P^{\prime}(n) \text { is dense in } R ; \\
& \bigcup_{n \in \omega} P^{\prime \prime}(n) \text { is dense in } R \text {. }
\end{aligned}
$$

Suppose further that $Q^{\prime}$ and $Q^{\prime \prime}$ are such sequences that, for $n \in \omega$ :

$$
\begin{aligned}
Q^{\prime}(n) & =\text { komponent }\left(R \sim P^{\prime}(n)\right) ; \\
Q^{\prime \prime}(n) & =\text { komponent }\left(R \sim P^{\prime \prime}(n)\right) .
\end{aligned}
$$

Assume:

$$
\begin{array}{cc}
\mathfrak{F}^{\prime}=\cup_{n \in \omega} Q^{\prime}(n) ; & \mathfrak{F}^{\prime \prime}=\bigcup_{n \in \omega} Q^{\prime \prime}(n) ; \\
A^{\prime}=\bigcap_{n \in \omega} \sigma Q^{\prime}(n) ; & A^{\prime \prime}=\bigcap_{n \in \omega} \sigma Q^{\prime \prime}(n) ; \\
\mathfrak{F}=\mathfrak{F}^{\prime} \cup \mathfrak{F}^{\prime \prime} ; &
\end{array}
$$


$\mu$ so measures $R$ that for $\beta \subset R, \mu(\beta)=0$ or $\infty$ according as $\beta$ is or is not countable.

With the help of .1 and 3.5.4 we infer:

$$
R=R \sim P^{\prime}(n) \cup R \sim P^{\prime \prime}(n) \subset \sigma Q^{\prime}(n) \cup \sigma Q^{\prime \prime}(n)=R
$$

for each $n \in \omega$. Consequently, in view of 3.6 we see that $A^{\prime} \cup A^{\prime \prime}=R$. Thus, because of $3.8 .7, .1,3.7,3.4$, and 3.3 we now know that: $\mathfrak{F}$ is Vitalian with respect to $A^{\prime} ; \mathfrak{F}$ is Vitalian with respect to $A^{\prime \prime}$; $\mathfrak{F}$ is not Vitalian with respect $A^{\prime} \cup A^{\prime \prime}$; moreover $R \sim \sigma(S)$ has the power of the continuum, and thus $\mu(R \sim \sigma(S)=\infty$, whenever $\$$ is a disjointed subfamily of $\mathfrak{F}$.

The above counterexample assures the invalidity of 1.10 . Notice that $\mathfrak{F}$ is countable and, in accordance with $3.9 \mathrm{M}$, covers $R$ in the sense of Vitali.

Now assume: $\delta=R, \rho$ so metrizes $R$ that

$$
\rho(x, y)=|x-y|
$$

for $x \in R, y \in R ; \phi \in \mathfrak{U}$ if and only if $\phi$ so measures $R$ that closed sets are $\phi$ measurable;

$$
V=\mathfrak{U E} \phi(\phi(\beta)<\infty \text { for each bounded subset } \beta \text { of } R) \text {. }
$$

If $\phi \in \mathcal{U}$ and $H$ is a $6.3 \mathrm{M}$ natural $6.2 \mathrm{M}$ blanket whose $2.2 \mathrm{M}^{\prime}$ spread is contained in $\mathfrak{N}$, then $H$ is a $\phi$ regular blanket as can be seen by taking $\Delta=\phi$ in $6.4 \mathrm{M}$. Thus according to $6.12 \mathrm{M}$, any natural blanket $H$, whose spread is contained in $\mathfrak{N}$, is $\phi$ strong whenever $\phi \in \mathcal{V}$, and is hence $2.7 \mathrm{M}^{\prime}$ perfect.

Let us agree here that $H$ is an absolutely perfect blanket if and only if corresponding to each $2.3 \mathrm{M}^{\prime}$ subblanket $H^{\prime}$ of $H$ there is a countable disjointed subfamily (s) of the spread of $H^{\prime}$ which covers all of the domain of $H^{\prime}$.

Clearly every absolutely perfect blanket is perfect. It is not hard to verify that every perfect blanket is natural and very easy to verify directly that absolutely perfect blankets are natural. If $H$ is any natural blanket whose spread is a net', then, because of 2.8, 2.13, $H$ is absolutely perfect. However if $H$ is any natural blanket whose domain includes a member of $\mathfrak{N}$ and whose spread is contained in $\mathfrak{N}$, then: $H$ is a perfect blanket; even though $H$ is thus $\phi$ strong for each $\phi$ in $V$ there 3.3 is nevertheless a $\phi$ in $\mathfrak{u}$, namely $\mu$, for which $H$ is not $\phi$ strong; $H$ is not absolutely perfect.

An easy consequence of 1.9 is the following:

.2 Theorem. If $\phi \in \mathcal{U}$ and if $H^{\prime}$ and $H^{\prime \prime}$ are $\phi$ strong blankets with 
domains disjoint and spreads contained in $\mathfrak{\complement}$, then $H^{\prime} \cup H^{\prime \prime}$ is a $\phi$ strong blanket.

In here forming $H^{\prime} \cup H^{\prime \prime}$ recall that $H^{\prime}$ and $H^{\prime \prime}$ are sets of ordered pairs.

From .2 we infer at once the following:

.3 Theorem. If $H^{\prime}$ and $H^{\prime \prime}$ are perfect blankets with domains disjoint and spreads contained in $\mathfrak{E}$, then $H^{\prime} \cup H^{\prime \prime}$ is a perfect blanket.

It is thus natural to conjecture the following:

.4 Statement. If $H^{\prime}$ and $H^{\prime \prime}$ are absolutely perfect blankets with domains disjoint and spreads contained in $\sqrt{ }$, then $H^{\prime} \cup H^{\prime \prime}$ is absolutely perfect.

A counterexample to .4 comes to hand as follows.

Suppose:

$$
B^{\prime}=A^{\prime} ; \quad B^{\prime \prime}=A^{\prime \prime} \sim A^{\prime} ;
$$

$H^{\prime}$ is such a function on $B^{\prime}$ that

$$
H^{\prime}(x)=\mathfrak{F}^{\prime} \mathrm{E} \beta(x \in \beta) \quad \text { for } x \in B^{\prime} ;
$$

$H^{\prime \prime}$ is such a function on $B^{\prime \prime}$ that

$$
H^{\prime \prime}(x)=F^{\prime \prime} \mathrm{E} \beta(x \in \beta) \quad \text { for } x \in B^{\prime \prime} .
$$

And note: $H^{\prime}$ and $H^{\prime \prime}$ are natural blankets with disjoint domains and spreads contained in the subset $\mathfrak{R}$ of $\mathfrak{E} ; H^{\prime}$ is an absolutely perfect blanket since its spread is contained in the net' $\mathfrak{F}^{\prime}$; similarly $H^{\prime \prime}$ is an absolutely perfect blanket; since the domain of $H^{\prime} \cup H^{\prime \prime}$ is $R$ and

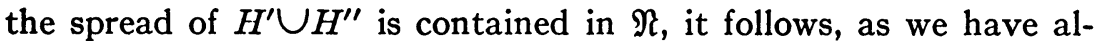
ready seen, that $H^{\prime} \cup H^{\prime \prime}$ is a perfect blanket which is neither absolutely perfect nor $\mu$ strong.

The University of California, Berkeley 